\title{
INVESTIGATIONS OF THIRD-HARMONIC GENERATION IN XENON BY MEANS OF A TOF MASS SPECTROMETER
}

\author{
L. MichalaK \\ Institute of Physics, Maria Curie-Sklodowska University \\ pl. Marii Curie-Sklodowskiej 1, 20-031 Lublin, Poland \\ AND R.J.J.M. STEENVOORDEN \\ FOM Institute for Atomic and Molecular Physics, Kruislaan 407, 1098 SJ Amsterdam \\ The Netherlands \\ (Received September 14, 1990; in final version May 10, 1991)

\begin{abstract}
TOF mass spectrometer for research of third-harmonic generation effect in isotropic media was used. The nonlinear medium in the experiment was Xe gas, the incident beam had the wavelength of $3546 \AA$ and the generated radiation had the wavelength of $1182 \AA$. A measure for the intensity of generated VUV was the intensity of the ion current generated at the spot where a non-homogeneous effusive molecular (2,2-dimethylbutane) beam was crossed by the VUV beam. The influence of parameters like the diameter of the UV beam and the Xe pressure on the VUV generation process was examined. The third-harmonic generation theory was used to verify the mass
\end{abstract} \\ spectrometric data.
}

PACS numbers: 42.65.Ky, 07.75.th

\section{Introduction}

The knowledge of characteristic of the VUV light intensity resulting from the third-harmonic generation process in the isotropic media is very important for many experiments utilizing this process. In a number of works [1-6] a vacuum monochromator and a solar blind photomultiplier were used for the investigations of VUV generation. In other works a vacuum monochromator and aceton-field ionization chamber [7] or nitricoxide photoionization chamber [8] were used. The 
aim of this work was to show the possibility of using an effusive molecular beam crossed with a focused photon beam, as an open ion source of TOF mass spectrometer for studying the third-harmonic generation in isotropic media. In this study the nonlinear medium was Xe gas. Xenon posesses both a nonlinear susceptibility and the proper dispersion characteristics to produce a negative value for the phase mismatch $\Delta k$. The incident beam had wavelength of $3546 \AA$, and the generated radiation had wavelength of $1182 \AA$. A measure for the intensity of the generated VUV was the intensity of the ion current generated at the spot where a non-homogeneous effusive molecular (2,2-dimethylbutane) beam was crossed by the VUV beam. The influence of parameters like the diameter of the UV (incident) beam and Xe pressure on the VUV generation process was examined. The TOF mass spectrometer was used to analyze and register the generated ions.

\section{Experimental}

The experimental set-up is shown in Fig. 1. The incident UV (3546 $\AA, 3.5 \mathrm{eV}$ - photon energy) pulsed beam was generated as a third-harmonic of Nd:YAG Laser. Diameter of this beam was defined by the diaphragm $D(2-7 \mathrm{~mm})$. The UV beam was focused in the center of the Xe cell $(L=200 \mathrm{~mm})$ by a quartz lens. $(f=200 \mathrm{~mm})$. In the focus of the primary beam the vacuum ultraviolet $(1182$ $\AA, 10.49 \mathrm{eV}$ - photon energy) was generated. The power density in the focus of the UV beam was varied by changing the size of the diaphragm $D$ and had values $1.4 \times 10^{11} \mathrm{~W} \mathrm{~cm}^{-2}$ and $1.4 \times 10^{12} \mathrm{~W} \mathrm{~cm}^{-2}$ for $D=2$ and $7 \mathrm{~mm}$ respectively. The pressure of Xe was monitored with a baratron. The LiF lens with the focal distance $f_{U V}=100 \mathrm{~mm}$ and $f_{V U V}=64 \mathrm{~mm}$ for UV and VUV respectively was placed after the Xe cell and focused the VUV beam inside the ionization chamber of mass spectrometer and at the same time formed the parallel UV beam. On the place of the VUV focus a non-homogeneous effusion molecular beam was generated by means of cylindrical capillary $70 \mathrm{~mm}$ long and $2 R=1 \mathrm{~mm}$ inner diameter (perpendicularly to drafts in Figs. 1(a),(b)). A measure of generated VUV intensity in the Xe cell was the number of 2,2-dimethylbutane $(10.06 \mathrm{eV}$ - ionization energy [9]) ions, which were generated in the intersection of the light and molecular beams and accelerated into the drift tube of the TOF mass spectrometer by an electric field. This system, where the effusive molecular beam is crossed with a photon beam just above the capillary outlet was the open ion source of this mass spectrometer. Using the non-homogeneous effusion molecular beam insured high density of molecules in the focus of VUV beam (higher numbers of produced photo-ions) [10-13]. Figure 2 shows the situation in the ion chamber of the mass spectrometer. The mass spectrum was recorded by using the channel plates detector and the LeCroy digital scope model 9400 with a sample rate of $100 \mathrm{MHz}$. Figure 3 shows the PIMS (Photolonization Mass Spectra) of 2,2-dimethylbutane obtained by using experimental set-up shown in Figs. 1 and 2. 
a)

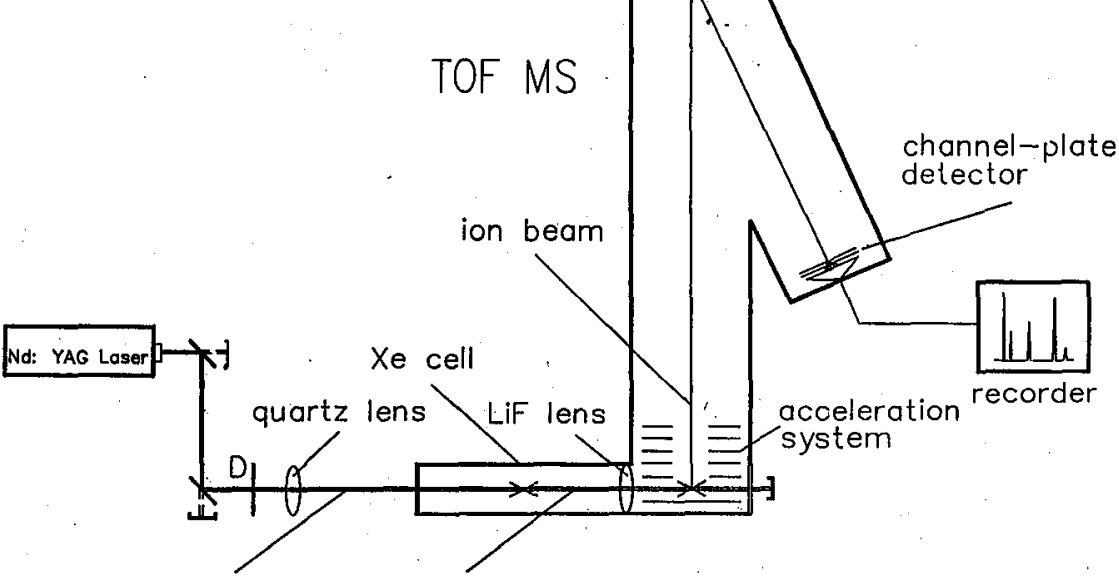

UV beam UV and VUV beams

b)

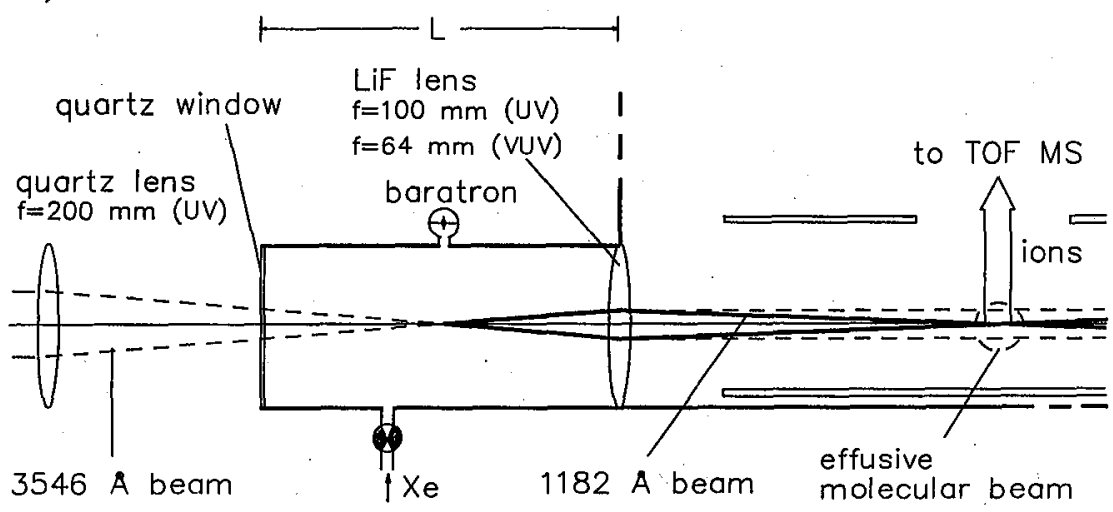

Fig. 1. (a) Experimental set-up used for investigating third-harmonic generation in xenon. The incident pulsed UV beam is generated as a third-harmonic of Nd:Yag Laser. A measure of the intensity of the generated VUV in the Xe cell is the intensity of the ion current from an ionization chamber TOF mass spectrometer. The PIMS (PhotoIonization Mass Spectra) was recorded by using a channel plates detector and oscilloscope. (b) The Xe cell and optical system used for generation and detection of the VUV beam. 


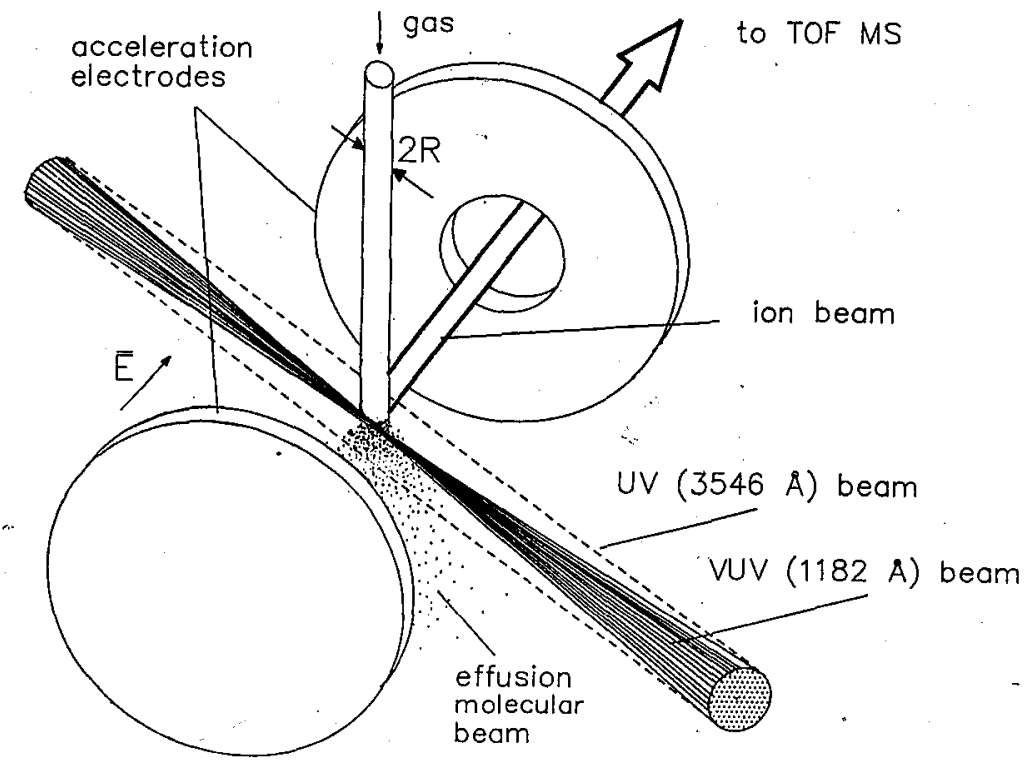

Fig. 2. The ionization chamber of the open ion source of TOF MS. The ions are generated in the intersection of the focused VUV and the effusive molecular beams.

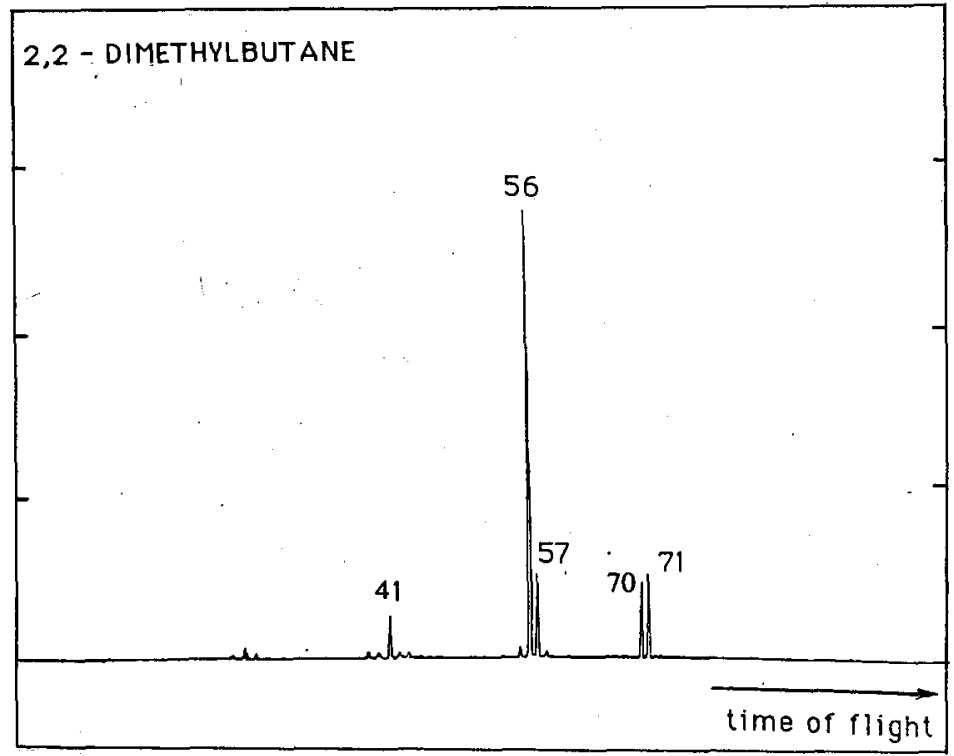

Fig. 3. Photoionization mass spectrum of 2,2-dimethylbutane obtained by using experimental set-up shown in Figs. 1 and 2. 


\section{Theory and calculations}

The experimental investigations presented in this work were verified using the third-harmonic generation process theory in the case of very tight focusing near the center of the gas cell $[14,15]$. It comes from this theory that the power $P_{3}$ of the generated VUV beam is given by:

$$
P_{3}=\frac{8.215 \times 10^{-2}}{\left(3 \lambda_{3}\right)^{4}}\left[\chi\left(\lambda_{3}\right)\right] N^{2} P_{1}^{3} F(b \Delta k, b / L, \delta / L),
$$

where $P_{1}$ and $P_{3}$ are the incident and generated powers in watts, $\lambda_{3}$ is the wavelength of the generated beam in $\mathrm{cm}$ (for our case $\lambda_{3}=1182 \AA$ ), $\chi\left(\lambda_{3}\right)$ is the third-order susceptibility at the third harmonic (for xenon $\chi(1182 \AA)=2.3 \times$ $10^{-35}$ esu [16]), $N$ is the number density of the nonlinear medium in $\mathrm{cm}^{-3}$, $F(b \Delta k, b / L, f / L)$ is a geometrical factor, $b$ is a confocal beam parameter, $L$ is the length of the cell which contained thenonlinear medium, $\int$ is the focal length of the lens used for focusing of the incident (UV) beam, $\Delta k$ is the phase mismatch defined by

$$
\Delta k=k_{3}-3 k_{1}
$$

where $k_{3} e_{4}=k_{1} e_{1}+k_{1} e_{2}+k_{1} e_{3}, k_{1} e_{i}$ and $k_{3} e_{4}$ are the wave vector of the incident radiation with wavelength $\lambda_{1}$ and the generated radiation with wavelength $\lambda_{3}$ respectively. The incident beam is focused, so that in general the three unit vectors $e_{i}$ come from three different directions and therefore $k_{3}$ is smaller than $3 k_{1}$.

The knowledge of the incident and generated powers is not necessary for calculations of the ratio $P_{3} / P_{1}^{3}$ but can be important for the interpretation of experimental results. In presented experiments, the pulsed (incident) UV laser beam had power $7 \times 10^{6} \mathrm{~W}$ for $7 \mathrm{~mm}$ (maximal diameter of UV beam used in our case) of the beam diameter. For smaller diameters of the UV beam this power is lower.

Figure 4 shows some of the variables used in our theoretical calculations of the ratio $P_{3} / P_{1}^{3}$. The incident beam was focused on an Xe cell of the length $L=200 \mathrm{~mm}$, with the beam waist of the radius $w_{0}$, being located at the center of the cell. The degree of focusing is characterized by the confocal beam parameter $b$ which is given by

$$
b=\left(2 \pi w_{0}^{2}\right) / \lambda_{1}=\left(2 \pi w_{0}^{2} n\right) / \lambda_{1,0},
$$

where $w_{0}=f \Theta$ (for small angles [17]), $\lambda_{1,0}$ is the vacuum wavelength, $n$ is the refractive index of gas at wavelength $\lambda_{1}$ (for $\mathrm{Xe}$ at $3546 \AA n \approx 1$ ). $\Theta$ is the far field diffraction half angle defined as

$$
\Theta=0.5 K\left(n_{1}\right)(D / f)^{3}+1.22 \lambda_{1} / n D,
$$

where $D$ is the diameter of the incident beam, $K\left(n_{1}\right)$ is an explicit function of the lens shape and $n_{1}$ is the refractive index of the lens. For our case, where quartz $\left(n_{1}=1.55\right)$ plano positive lens was used, $K\left(n_{1}\right)$ is given by Eq. (5) [17]:

$$
K\left(n_{1}\right)=\frac{1}{32}\left(n_{1}-1\right)^{-2}\left(n_{1}^{2}-2 n_{1}+\frac{2}{n_{1}}\right) .
$$




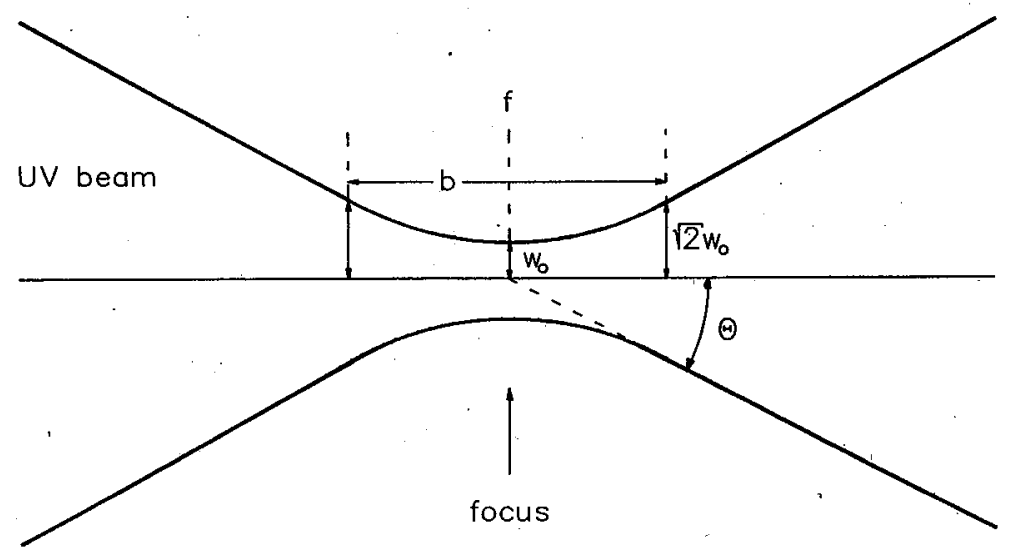

Fig. 4. The focusing scheme. Marked are variables used for calculations $P_{3} / P_{1}^{3}$ in this work: $b$ - confocal beam parameter, $w_{0}$ - radius of the beam waist, $\Theta$ - the far field diffraction angle.

In the tight focusing case $(b / L \approx 0)$ the geometrical factor $F$ has the following form [14]:

$$
F(b \Delta k, 0,0.5)= \begin{cases}\pi^{2}(b \Delta k)^{2} \exp (b \Delta k / 2) & \text { for } \Delta k \leq 0 \\ 0 & \text { for } \Delta k \geq 0\end{cases}
$$

In presented experiments $b / L$ is 0.14 and 0.012 for $D=2 \mathrm{~mm}$ and $D=$ $7 \mathrm{~mm}$ respectively but $F$ differs not much for $b / L=0$ [14]. The function $F$ has a maximum for $b \Delta k=-2$. This can be obtained by changing the density $N$ of the Xe gas because $\Delta k=N C(\lambda)[15]$, where $C(\lambda)$ is the per atom dispersion (for Xenon $\left.C(1182 \AA)=-5.99 \times 10^{-17} \mathrm{~cm}^{2}\right)$. From the Eq. (1) using Eqs. (3-6) we have calculated the ratio $P_{3} / P_{1}^{3}$ for certain diameters $(D=2,3,4,5,6$ and $7 \mathrm{~mm})$ of the incident ( $3546 \AA$ ) beam as a function of Xe pressure in the gas cell.

\section{Results}

In Figs. 5(a), (b) the intensities of 2,2-dimethylbutane ion currents (normalized on the 3rd power of the incident radiation in the focus) as a function of Xe pressure in the Xe cell (the place of VUV generation) for several values of the diameter $D$ of UV beam are compared with the theoretical values $P_{3} / P_{1}^{3}$ (see Eq. 1). Figure 5(a) shows this comparison for $D=5,6$ and $7 \mathrm{~mm}$ and additionally experimental results for $D=2,3$ and $4 \mathrm{~mm}$. The comparison of experimental and theoretical results for $D=2,3$ and $4 \mathrm{~mm}$ is presented in Fig. 5(b). The good agreement of experimental data and theoretical calculations is evident. The limit of $7 \mathrm{~mm}$ for the maximum diaphragm is determined by the diameter of the Nd:YAG beam. The asymmetry in the curves is due to the asymmetry in the function $F$ (Eq. 1). The larger the angles between the three wave vectors, the larger must be 

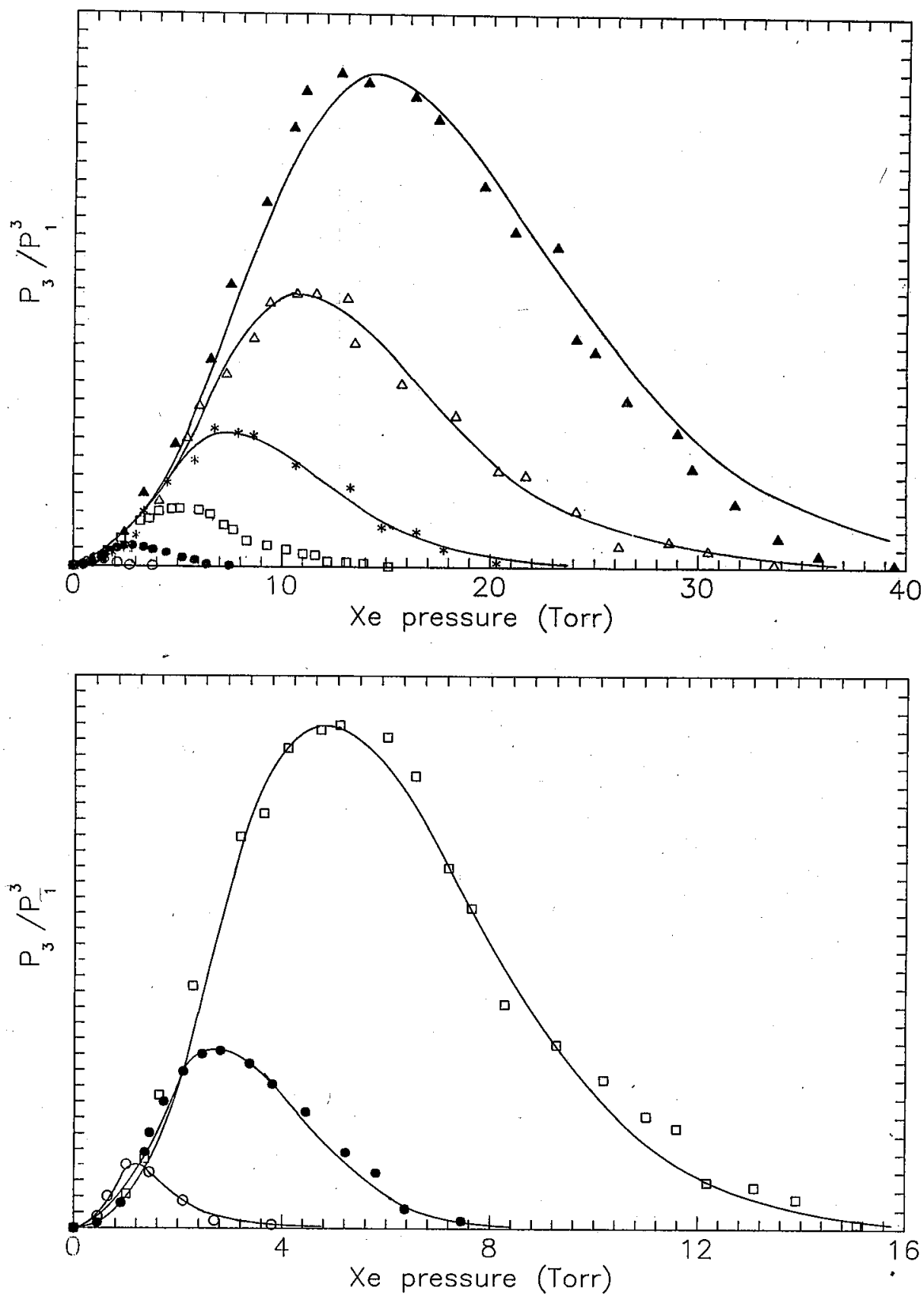

Fig. 5. Conversion efficiency $\left(P_{3} / P_{1}^{3}\right)$ of thie third-harmonic generation as a function of Xe pressure in the Xe cell. (a) Diameter of UV (3546 $\AA$ ) beam $D=2(0), 3(\bullet), 4$ (), $5(*), 6(\Delta)$ and $7 \mathrm{~mm}$ (full $\triangle$ ). (b) $D=2(0), 3(\bullet)$ and $4 \mathrm{~mm}(\square)$; in this case the axis of pressure ranges from 0 to 16 Torr (cl. Fig. 5(a)). (-) theoretical curve calculated from Eq. 1. 
the difference in refractive indices to have momentum conservation. Therefore the optimum pressure increases with the size of the diaphragm.

In Fig. 6 the maxima of the curves in Figs. 5(a); (b) are compared with $P_{3} / P_{1}^{3}$ as a function of $\mathrm{Xe}$ pressure. For this, function $F$ is replaced by its maximum value.

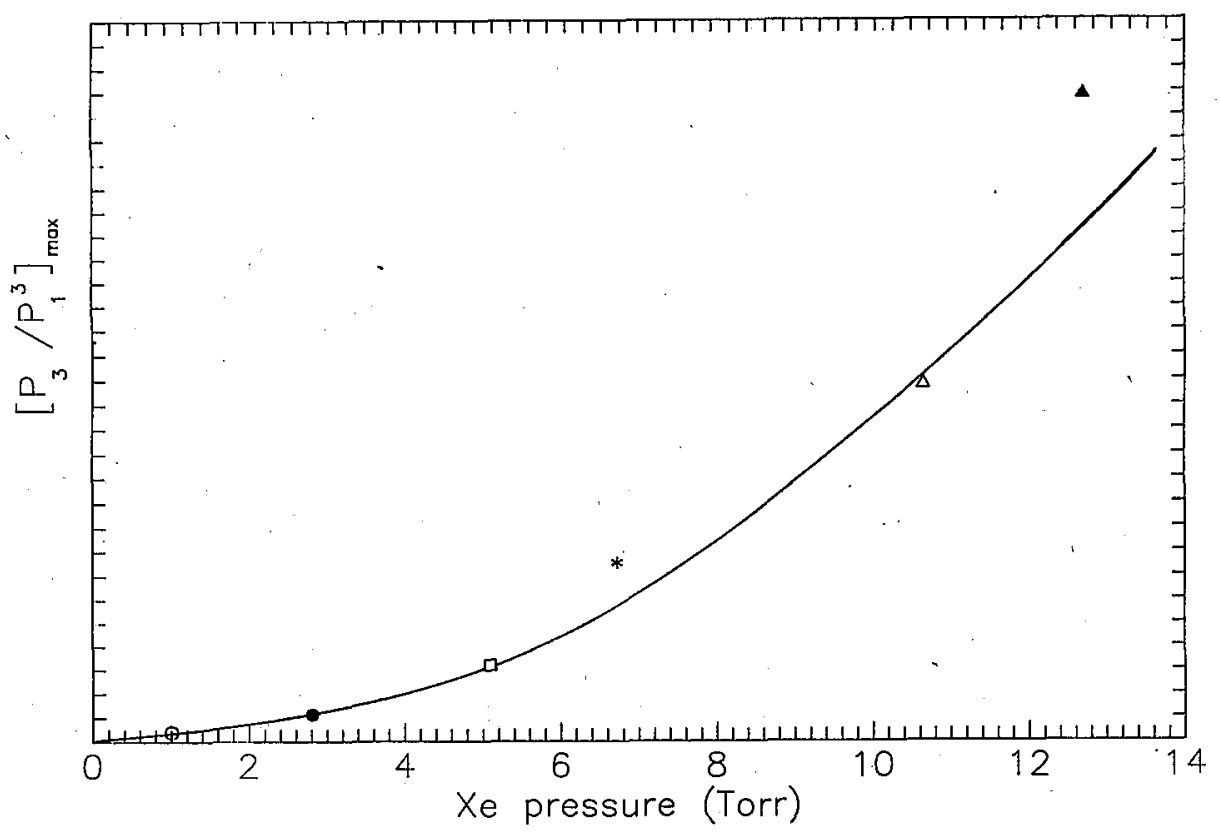

Fig. 6. Maximum conversion efficiency $\left(P_{3} / P_{1}^{3}\right)_{\max }$ as a function of Xe pressure in the Xe cell. Diameter of UV $(3546 \Omega)$ beam $D=2(\circ), 3(\bullet), 4(\square), 5(*), 6(\triangle)$ and $7 \mathrm{~mm}$ (full $\triangle$ ); $(-)$ theoretical curve calculated from Eq. 1.

The measurements of an ion current corresponding to an intensity of VUV radiation were accompanied by an experimental error of at least $\pm 5 \%$ (not marked in Figs. 5(a), (b) and 6).

An increase in incident power (increase of the diameter of incident beam) will not give an increase in generated power anymore. Several authors have reported experiments in which limitations were observed in nonresonant harmonic conversion in rare gases [18]. Zych and Young [16] observed that the conversion efficiency for tightly focused beams in pure Xe reached a constant value of about $2 \times 10^{-4}$, becoming independent of the pump power for pump intensities above $5 \times 10^{12} \mathrm{~W} \mathrm{~cm}^{-2}$. In our experiments the maximum value of the power density in the focus of the UV beam is $1.4 \times 10^{12}$. Also an increase in xenon density does not infinitely increase the maximum conversion efficiency as Eq. (1) might suggest. Several processes can limit the conversion efficiency, as there are linear and nonlinear absorption, multi-photon ionization, dielectric breakdown, Stark shift and the Kerr effect. These are extensively discussed in [18]. 


\section{Conclusion}

In this work, the system of crossing a non-homogeneous molecular beam with a focused VUV beam in an open ion source of a TOF mass spectrometer wasused to investigate the third-harmonic generation in xenon. The diameter influence of the incident beam and the Xe pressure on the characteristic of generation of the VUV beam was obtained. The good agreement ofexperimental results with the third-harmonic theory shows that the method used in this work appears to be very useful.

\section{Acknowledgements}

The authors wish to thank Dr. P.G. Kistemaker and Dr. A.E. de Vries for their useful suggestions and cooperation. This work is a part of the research programme of the Dutch Foundation for Fundamental Research on Matter (FOM) and was made possible by financial support from the Netherlands Organization for the Advancement of Pure Research.

\section{References}

[1] A.II. Kung, Appl. Phys. Lelt. 29, 653 (1974).

[2] D.M. Bloom, J.T. Yardley, J.F. Young, S.E. Harris, Appl. Phys. Lett. 24, 427 (1977).

[3] A.II. Kung, J.F. Young, S.E. II arris, Phys. Rev. Lell. 29, 985 (1972).

[4] Yun-Mui, K.D. Bonin, T.J. McIlorth, Opt. Letl. 7, 268 (1982).

[5] D. Cotter, Opt. Commun. 31, 379 (1979).

[6] M.N.R. Ashfold, C.D. IIeryet, I.D. Prince, B. Tutcher, Chem. Phys. Letl. 131, 291 (1986).

[7] A.II. Kung, Opl. Lell. 8, 24 (1983).

[8] D. Cotter, Opl. Letl. 4, 134 (1979):

[9] H. Jupnik, Phys. Rev. 60, 884 (1941).

[10] B. Adamczyk, L. Michalak, Int. J. Mass Spectrom. Ion Process. 69, 163 (1986).

[11] B. Adamczyk, L. Michalak, Int. J. Mass Spectrom. Ion Process. 71, 211 (1986).

[1.2] B. Adamczyk, L. Michalak, Inl. J. Mass Speclrom. Ion Process. 74, 235 (1986).

[13] L. Michalak, B. Adamczyk, Inl. J. Mass Spectrom. Ion Process. 85, 319 (1988).

[14] G.C. Bjorklund, IEEE J. Quanlum Eleclron. QE-11, 287 (1975).

[15] R. Manhon, T.J. McIlorth, V.P. Myerscough,D.W. Koopman, IEEE J. Quanlum Electron. QE-15, 144 (1979).

[16] L.J. Zych, J.F. Young, IEEE J. Quanlum Electron. QE-14, 147 (1978). 
[17] Optics Guide, Melles Griot, No 3, 36 (1985).

[18] J.F. Reintjes, Nonlinear Optical Parametric Processes in Liquides and Gases, Chap. 4, Academic Press, New York 1984. 